\title{
THE UIT SURVEY OF THE ULTRAVIOLET SKY BACKGROUND
}

\author{
W.H. WALLER ${ }^{1,2}$ AND T.P. Stecher ${ }^{2}$ \\ ${ }^{1}$ Hughes STX \\ ${ }^{2}$ NASA Goddard Space Flight Center \\ AND
}

The Ultraviolet Imaging Telescope Science Team

http://fondue.gsfc.nasa.gov/UIT/UIT_HomePage.html

\section{Introduction}

When viewed from above the Earth's atmosphere, the nighttime ultraviolet sky background is profoundly dark. Recent measurements indicate that the diffuse UV sky background is up to 100 times (5 magnitudes) fainter than the equivalent visible background as measured from the ground. Much of this difference can be attributed to the Sun's lower emissivity at UV wavelengths, leading to reduced irradiation of and scattering by the interplanetary dust. Because the resulting Zodiacal light is so much weaker in the $\mathrm{UV}$, a comprehensive characterization of the UV sky can yield important information on the more distant Galactic and extragalactic backgrounds and, ultimately, on their material origins (see Brosch, this volume, p. 57).

Despite recent concerted efforts, the strength and spatial distribution of the UV background remain controversial topics. Estimates range from a few hundred photons $\mathrm{s}^{-1} \mathrm{~cm}^{-2} \mathrm{sr}^{-1} \AA^{-1}\left(\sim 27 \mathrm{mag} \operatorname{arcsec}^{-2}\right)$ with no obvious spatial distribution to several thousand "photon units" with a strong gradient toward the Galactic midplane (cf. Bowyer 1991; Henry 1991). Models of the stellar UV radiation field depend critically on the distribution and scattering properties of the interstellar dust, yielding significantly different predictions as a function of grain albedo and phase function (cf. Murthy \& Henry 1995). Herein, we summarize recent results from an analysis of UV images obtained by the Ultraviolet Imaging Telescope (Waller et al. 1995). 


\section{The UIT Experiment}

As part of the December 1990 Astro-1 Spacelab mission on the Space Shuttle Columbia, the Ultraviolet Imaging Telescope (UIT) obtained 361 NUV $(\sim 2500 \AA)$ images and $460(\sim 1500 \AA)$ images of the sky in 66 separate pointings - each with a 40 arcmin field of view and a resolution of $\sim 2$ arcsec. The resulting images enable discrimination between the diffuse sky and discrete objects (stars, nebulae, and galaxies with $\mathrm{m}_{U V}<20$ mags) for true background measurements. Another 78 target fields were imaged in the FUV during the March 1995 Astro-2 mission. Processing and calibration of the Astro-2/UIT images is nearing completion.

The UIT shared the Spacelab's Instrument Pointing System with the Hopkins Ultraviolet Telescope (HUT) and the Wisconsin Ultraviolet PhotoPolarimeter Experiment (WUPPE). Simultaneous spectral observations by the HUT could be compared with UIT's FUV backgrounds, thereby constraining the effects of atmospheric nightglow on UIT's FUV backgrounds.

\subsection{EFFECTS OF AIRGLOW}

Photometric analysis of the UIT images has yielded positive detections of FUV and NUV backgrounds in both the daytime and nighttime skies. The FUV backgrounds are dominated by the effects of airglow-even at nightcorrelating with the OI $(\lambda \lambda 1304,1356)$ line emission measured by HUT. An excess background of roughly 700 photon units $\left(\sim 26 \mathrm{mag} \operatorname{arcsec}^{-2}\right)$ indicates Galactic and extragalactic contributions. Total background intensities similar to this excess are found in the deep FUV-B1 images that were obtained during the March 1995 Astro-2 mission-a time when the Sun was at minimum activity, producing airglow levels $\sim 3$ times lower than experienced during the Astro-1 mission.

\subsection{EFFECTS OF STRAY LIGHT}

Stray light from the Sun dominates the daytime NUV backgrounds, while stray light from UV-bright stars (just beyond the field of view) can occasionally produce an appreciable effect at night. The resulting backgrounds can be fit by a single "Baffle Function." The Moon's deviation from this fit indicates that stray Moonlight is a negligible contributor to the measured backgrounds.

\subsection{ZODIACAL BACKGROUNDS}

Away from the Galactic plane, the nighttime NUV backgrounds are correlated at the $98 \%$ confidence level with the Zodiacal UV light predicted 
from visible-light measurements-both backgrounds decreasing with ecliptic latitude. These relations are best fit with a NUV/VIS "color" of $0.5 \pm$ 0.2 (where the solar emissivity spectrum gives a color of unity) and with an extrasolar component at high galactic latitude of $300 \pm 300$ photon units.

\subsection{GALACTIC BACKGROUNDS}

Both the FUV and NUV intensities show strong dependences on Galactic longitude and latitude, reaching the highest levels in diffuse regions next to structured nebulosity (e.g., Cygnus, Vela, and Gum fields). The blue $(F U V-N U V)$ colors at these high levels $\left(\sim 10^{4}\right.$ photon units) are consistent with scattering of ambient OB starlight by galactic dust. The location of the dust is uncertain, but is probably associated with the adjoining nebulosity.

\subsection{EXTRA-GALACTIC BACKGROUNDS}

The nighttime NUV intensities - after subtraction of a Zodiacal component with a NUV/Vis color of $0.5 \pm 0.2$-yield residual intensities that correlate with FIR measurements of the corresponding fields. Extrapolation of this NUV-FIR relation to negligible FIR intensities indicates an extragalactic NUV emission component of $200 \pm 100$ photon units. Such an estimate for the "cosmic" UV background supports the low intensities that have been proposed in the debate over the strength and structure of the UV background (cf. Henry 1991; Bowyer 1991; Brosch, this volume, p. 57).

\section{Conclusions}

By imaging in selected UV "windows" that do not include the OI airglow emission or significant Zodiacal light, one can reduce the sky background to 300 photon units $\left(27 \mathrm{mag} \operatorname{arcsec}^{-2}\right)$ or fainter. Such a dark sky is ideal for pursuing studies of the dim outer regions of nearby galaxies, lowsurface-brightness galaxies within the local supercluster, as well as faint primeval galaxies much farther away (O'Connell 1987). Ultraviolet imaging experiments such as the wide-field UIT and narrow-field HST/WFPC2 and HST/FOC cameras can benefit from the darker skies and subsequently enhanced contrasts that are available in these UV "windows." Future surveys with more sensitive UV detectors than are currently available should be able to more fully characterize and exploit the dark-sky advantage that UV imaging affords (cf. Brosch, this volume, p. 57). 


\section{Acknowledgements}

UIT research is funded through the Spacelab Office at NASA Headquarters under Project number 440-51.

\section{References}

Bowyer, S. 1991, Ann.Rev.Astron.Astrophys., 29, 59

Brosch, N., this volume, 57

Henry, R. C. 1991, Ann.Rev.Astron.Astrophys., 29, 89

Murthy, J., \& Henry, R. C. 1995, Astrophys.J., 448, 848

O'Connell, R. W. 1987, Astron.J., 94, 876

Waller, W. H., et al. 1995, Astron.J., 110, 1255 